
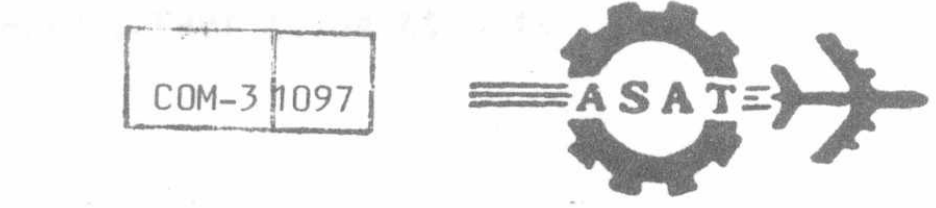

MILITARY TECHNICAL COLLEGE

CAIRO - EGYPT

\title{
A SELF ADAPTIVE EQUALIZER WITH RAPID ACQUISITION
}

Conventional adaptive equalization of data transmission channels requires an initial training period during which a known data sequence is transmitted and properly synchronized at the receiver. Transmission of the training sequence is not convenient for multipoint data communication networks. Recently, research is directed towards equalization schemes that operate without initial training sequence. Such schemes are called self-adaptive equalizers(SAE). Most of SAE schemes available in literature need a long time to converge to the optimum equalizer.

In this paper we propose an equalization scheme that shortens this time significantly. The proposed scheme is a cascade of two adaptive equalizers. The first one is short and thus it converges rapidly. The partial equalization done by this equalizer is coarse and will not enable reliable recovery of data. The second equalizer is long and it starts adaptation after the convergence of the short one. Due to the partial equalization performed by this latter the convergence time decreases. The length of the long equalizer is determined by the desired precision of equalization. This scheme is simulated on a digital computer. Simulation results exhibit superiority of the proposed scheme over published ones.

\section{INTRODUCTION}

A great deal of research has been performed in the last twenty years for equalizing data transmission channels [e.g. 1-3]. Equalization is done by passing samples of received signal through an adaptive equalizer consisting of tapped delay line having adjustable coefficients (tap-weights). The equalizer tapweights are adjusted by starting the transmission with a short training sequence of digital data which is known in advance by

Ass. Prof., Chair of Electronic Warefare, M.T.C.

Ass. Prof., Chair of Comm., M.T.C.

t* Eng., M.Sc., Air Defence College. 
the receiver. This mode is called the training mode. At the end of this mode the equalizer coefficients reach their optimum values. Thenceforward the equalizer will use the detected data instead of actual data to continue the adaptation process. This latter mode is called the decision-directed mode, and it is intended for tracking possible changes in channel characteristics during transmission.

In modern multipoint data-communication netweorks, several receivers may be hearing the same transmitter at the same time. The problem pecular to multipoint networks is that of retraining a tributary receiver which, because of drastic changes in channel characteristics or simply because it was not powered-on during initial network synchronization, is not able to recognize data and polling messages. Since lines are shared, the control modem has to interrupt data transmission and initiate a new synchronization procedure generally causing all tributaries to retrain.

It is clear that, particularly for large or heavily loaded multipoint networks, data throughput is increased and network monitoring is made easier by giving tributary receivers the capability to achieve complete adaptation without disrupting normal data transmission to other terminals. The equalizers used to make the receivers operating as explained above are called self adaptive equalizers (SAE).

A common feature of previous SAE algorithms [3-7] is that they need a relatively long time to converge to the optimum setting. This arawback may be not tolerable in cases of rapidiy varying channels. Also, the information lost during this time will be large. In this paper, we propose an SAE algorithm that has rapid convergence and low steady state error. The proposed scheme is a cascade of two adaptive equalizers. The first one is short and thus it converges rapidly. The second equalizer is long and it starts adaptation after the convergence of the short one. Due to the partial equalization performed by this latter, the convergence time of the long equalizer is reduced.

The paper is orgainzed as follows : section II gives a review on the previous work in the domain of SAE. The proposed SAE is given in section III, and its simulation results are given in section IV. Finally section $V$ is devoted to discussion of results and conclusions.

\section{REVIEW ON PREVIOUS WORK}

The block diagram of data trarsmission system is given in Fig.l, in which the "channel "includes the effects of the transmitter filter, the modulator, the transmission medium, and the demodulator. A symbol $a_{k}$ which is one of $\mathrm{L}$ discrete amplitude levels, is the transmitted data at instant $\mathrm{kT}$, where $\mathrm{T}$ seconds is the signaling inlerval. Let the vector $\mathrm{X}_{\mathrm{k}}$ represent the $\mathrm{N}$ samples measurments that are available at the channel output and have 
some relationship with $a_{k}$.

The equalizer consists of a tapped-delay line having $\mathrm{N}$ adjustable coefficients (tap-weights). Let $\mathrm{H}$ denote the vector composed of the equalizer coefficients. The output of the equalizer $y_{k}=H^{\top} x_{k^{\prime}} H^{\top}$ is the transpose of $\mathrm{H}$, is a linear estimate of $a_{k}$ of the basis of $x_{k}$. The optimum equalization vector $H_{k}$ is the one that minimizes the difference between $y_{k}$ and $a_{k}$ according to some criterion. According to the popular minlmum mean-square error criterion, $\mathrm{H}_{*}$ is the vector that minimizes $\mathrm{E}\left(\left(\mathrm{a}_{\mathrm{k}}-\mathrm{y}_{\mathrm{k}}\right)^{2}\right)$.
This value is the solution of the Wiener-Hoph equation,

where;

$$
\mathrm{RH}_{*}=\mathrm{P}
$$

$$
R=E\left(x_{k} x_{k}^{T}\right), \quad P=E\left(a_{k} x_{k}\right)
$$

Direct solution of this equation implies matrix inversion which is a complicated process that is usually avoided in signal processing. In practice, this solution is searched iteratively with the help of the least-mean square (LMS) algorithm of Widrow,

$$
\mathrm{H}_{\mathrm{k}+1}=\mathrm{H}_{\mathrm{k}}+\mu\left(\mathrm{a}_{\mathrm{k}}-\mathrm{H}_{\mathrm{k}}^{\mathrm{T}} \mathrm{x}_{\mathrm{k}}\right) \mathrm{x}_{\mathrm{k}}
$$

where $\mu$, called the step size, is a small positive constant that controls the convergence of the algorithm (2). This algorithm is used in the training mode. At the end of this mode, the equalizer coefficients reach their optimum values, $H_{\star}$. Then the adaptation is continued by recovered data $\hat{a}_{k}$ instead of $a_{k}$ to track any, possible, variations in channel characteristics. Thus the adaptation is continued according to the decision directed algorithm,

$$
\mathrm{H}_{\mathrm{k}+1}=\mathrm{H}_{\mathrm{k}}+\mu\left(\hat{\mathrm{a}}_{\mathrm{k}}-\mathrm{H}_{\mathrm{k}}^{\mathrm{T}} \mathrm{x}_{\mathrm{k}}\right) \mathrm{x}_{\mathrm{k}}
$$

where;

$$
\hat{a}_{\mathrm{k}}=\operatorname{dec}\left(\mathrm{y}_{\mathrm{k}}\right)
$$

$\hat{a}_{k}$ is obtained by quantizing $y_{k}$ to the nearest data level.

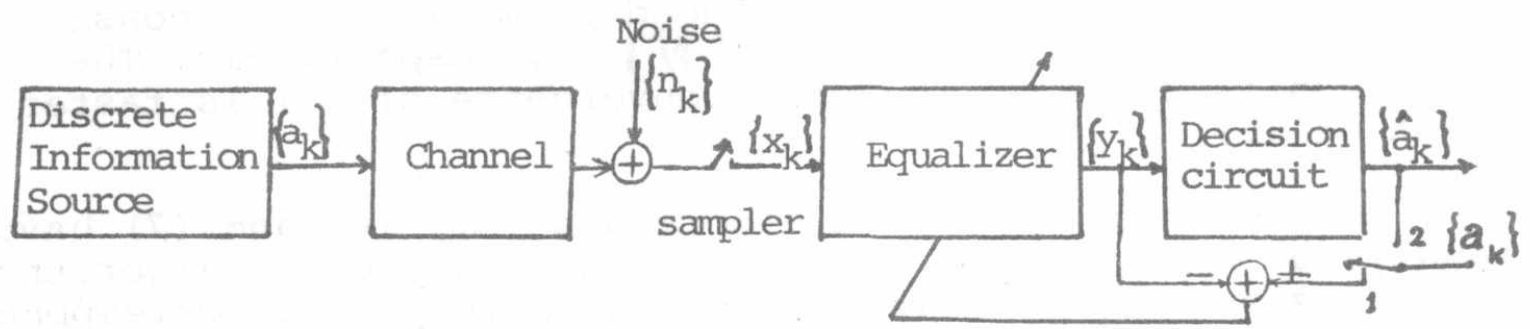

Fig.1. Block diagram of a data transmission system. 
SAE schemes do not have a training mode. The direct use of the algorithm (3), from the start of communication, succeeds in reaching near to $\mathrm{H}_{*}$ for the cases where the eye pattern is initially open. This is the case of channels with mild distortion. If the eye pattern is initially closed, the algorithm (3) may diverge as has been observed from simulation and experimental results of this algorithm.

To overcome this problem, Sato[3]presented the following SAE algorithm,

$$
\mathrm{H}_{\mathrm{k}+1}=\mathrm{H}_{\mathrm{k}}+\mu\left(\gamma_{\mathrm{sign}}\left(\mathrm{H}_{\mathrm{k}}^{\mathrm{T}} \mathrm{x}_{\mathrm{k}}\right)-\mathrm{H}_{\mathrm{k}}{ }^{\mathrm{T}} \mathrm{x}_{\mathrm{k}}\right) \mathrm{x}_{\mathrm{k}^{\prime}}, \gamma=\frac{\mathrm{E}\left(\mathrm{a}_{\mathrm{k}}^{2}\right)}{\mathrm{E}\left(\left|\mathrm{a}_{\mathrm{k}}\right|\right)}
$$

The probability of an erroneous sign-decision is smaller than that of the direct multilevel decision (3), (4). This explains the superiority of Sato algorithm (5) to the decision directed one $(3),(4)$.

The disadvantage of Sato algorithm is that its equalization is more coarse than that of the decision directed algorithr. This problem is overcome by using Sato algorithm to open the eye pattern and then adaptation is switched to the decision directed algorithm (3), (4).

In [4] two other SAE algorithms, for quadrature amplitude modulation data communication systems, are presented. In such systems $a_{k^{\prime}} x_{k}$ and $H_{k}$ are complex quantities. Denoting by $x^{k}$ the complex conjugate of $\mathrm{x}_{\mathrm{k}}$, those algorithms are :

$$
\mathrm{H}_{\mathrm{k}+1}=\mathrm{H}_{\mathrm{k}}+\mu \mathrm{y}_{\mathrm{k}}\left(\frac{\alpha}{\left|\mathrm{y}_{\mathrm{k}}\right|}-1\right) \mathrm{x}_{\mathrm{k}}^{*} ; \alpha=\frac{\mathrm{E}\left(\left|\mathrm{a}_{\mathrm{k}}\right|^{2}\right)}{\mathrm{E}\left(\left|\mathrm{a}_{\mathrm{k}}\right|\right)}
$$

and

$$
\mathrm{H}_{\mathrm{k}+1}=\mathrm{H}_{\mathrm{k}}+\mu_{\mathrm{y}_{\mathrm{k}}}\left(\beta-\left|\mathrm{y}_{\mathrm{k}}\right|^{2}\right) \mathrm{x}_{\mathrm{k}}^{*} ; \beta=\frac{E\left(\left|\mathrm{a}_{\mathrm{k}}\right|^{4}\right)}{E\left(\left|\mathrm{a}_{\mathrm{k}}\right|^{2}\right)}
$$

The algorithms (6) and (7) are intended to minimize the coast functions, $E\left[\left(\left|y_{k}\right|-\alpha\right)^{2}\right]$ and $E\left[\left(\left|y_{k}\right|^{2}-\beta\right)^{2}\right]$, respectively. The results given in [4] show that the convergence of (7) is faster than that of $(6)$.

In [5-7], the convergence of SAE algorithms (5), (6) and (7) have been analyzed. According to those papers, the above SAE algorithms have numerous stationary points, some of those points correspond to good equalizers, while others correspond to bad ones. The points to which the algorithm converges depend on the initial setting of the equalizer. Thus, in general, one does not know if the above SAE algorithms will converge to a good equalizer or to a bad one. However, simulation results, given in [4], show that 
in cases of channels with mild distortion, the above algorithms will converge to a good equalizer if one of the tap-weights is initially set to a large value, while the remaining ones are set to small (or zero) values.

\section{PROPOSED SAE SCHEME}

The proposed equalization scheme,Fig.2, is a cascade of two transversal filters. The first filter is short, i.e. it has small number of taps $\mathrm{N}_{1}$, while the second one is long, it has $\mathrm{N}_{2}$ taps. The principle of operation of this scheme relies on the following facts known from the theory of adaptive equalization [9].

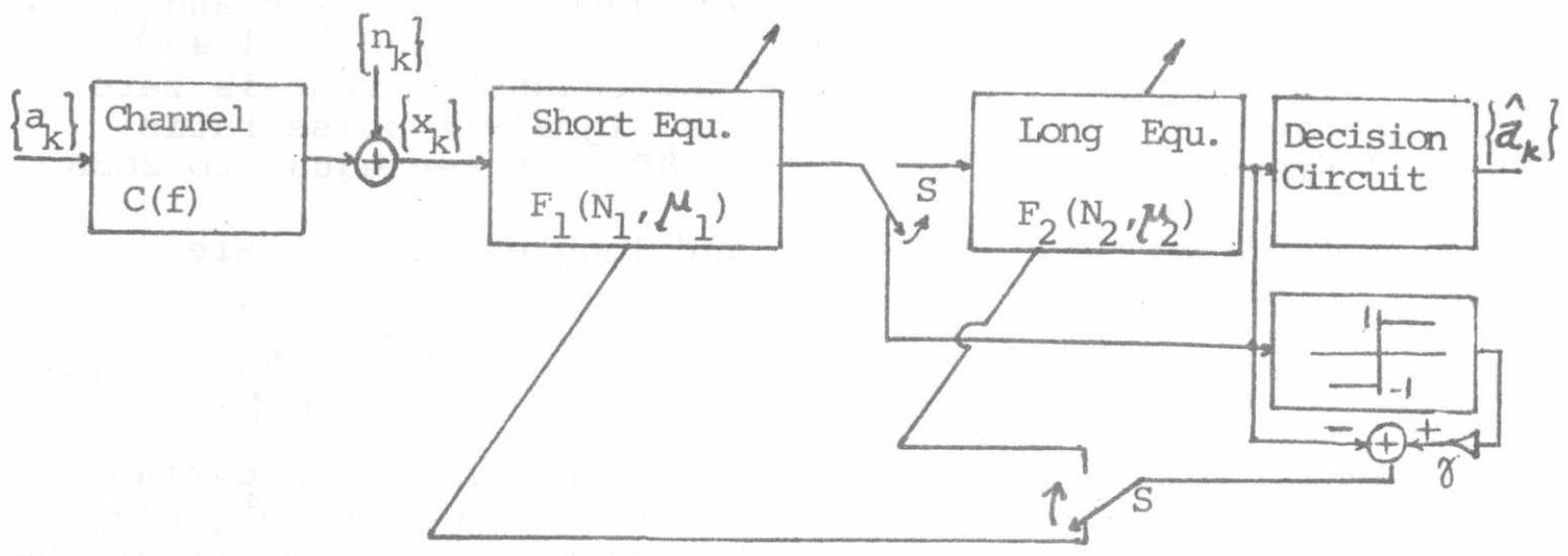

Fig. 2. SAE using two stages transversal filters.

As the equalizer length $\mathrm{N}$ increases, equalization becomes more precise. However, the allowable step size $\mu$ decreases and, consequently, the convergence speed decreases. The greater is the level of noise in the control error signal $\left(a_{k}-y_{k}\right.$ in(3) and $\gamma$ sign $\left(y_{k}\right)-y_{k}$ in (5)) the smaller should be the step sizie; thus the slower will be the convergence. Finally for a given adaptation algorithm (i.e. given $N$ and $\mu$ ), the farer is the initial setting from $\mathrm{H}_{\star}$, the longer will be the convergence time. The above arguments imply that precise $\mathrm{SAE}$ needs a long time to converge.

Now, in the proposed scheme, the short equalizer ensures rapid convergence. The partial equalization offered by this equalizer makes the equivalent channel in front of the long equalizer a mild one. This improves both the speed and accuracy of convergence of the long equalizer.

The adaptation of the proposed equalizer will be done in the following way. On the start of the adaptation process, only the coefficients of the short equalizer are adaptive. After the convergence of the short equalizer has been settled (say after ie samples) the adaptation of the long equalizer is started while the adaptation of the short equalizer is terminated. The reason for this termination is to protect the long equalizer from the adaptation noise of the short one. 


\section{SIMULATION RESULTS}

Intersymbol interference equalization can be carried out for all pulse-modulation systems, including pulse amplitude medulation (PAM), frequency shift-keying, phase shift-keying and quadrature amplitude modulation.

In this paper only PAM systems are considered in the simulations. Conclusions, however, can be easily extended to other types of modulation. Impulse response and sampled impulse response of a typical telephone channel are given in figures 3 and 4 [5]. The data to be transmitted are equally distributed over L possible values. We have considered two cases. Those are $: L=8$ and $L=4$ and the data alphabets are $\{ \pm 1, \pm 3, \pm 5, \pm 7\}$ and $\{ \pm 1, \pm 3\}$ respectively. The additive noise at the channel output is zero mean white Gaussion with power $\sigma^{2}$ The signal-to-noise ratio (SNR) is defined as SNR $=\mathrm{E}\left(\mathrm{a}_{\mathrm{k}}^{2}\right) / \mathrm{\sigma}^{2}$. SNR is taken equal to $20 \mathrm{OB}$.

The coefficients of both the short and long equalizers are apdated according to the algorithm,

$$
\mathrm{H}_{\mathrm{k}+1}=\mathrm{H}_{\mathrm{k}}+\mu\left(\gamma \operatorname{sign}\left(\mathrm{H}_{\mathrm{k}}^{\mathrm{T}} \mathrm{x}_{\mathrm{k}}\right)-\mathrm{H}_{\mathrm{k}}{ }^{\mathrm{T}} \mathrm{x}_{\mathrm{k}}\right) \mathrm{x}_{\mathrm{k}}, \gamma=\frac{\mathrm{E}\left(\mathrm{a}_{\mathrm{k}}^{2}\right)}{\mathrm{E}\left(\mathrm{a}_{\mathrm{k}}\right)}
$$
Before we give simulation results, it is convenient to define
the following quantities. The first is the data power $a^{2}=E\left(a_{k}^{2}\right)$. The second is the instantenous mean-square error $\mathrm{E}\left(\left(\mathrm{a}_{\mathrm{k}}-\mathrm{y}_{\mathrm{k}}\right)^{2}\right)$ at $\mathrm{t}^{\mathrm{k}}$ the output of the equalizer. Its estimate $\varepsilon_{n}$ is calculated over the interval $[(n-1) M+1, n M]$ according to

$$
\epsilon_{n}=\frac{\sum_{i=(n-1) M+1}^{n M}\left(a_{i}-y_{i}\right)^{2}}{M}, n=1,2, \ldots
$$

where; $M$ is few tens of samples. ( $M=100$ samples). The third is the error rate $P_{n}$ in the interval $[(n-1) M+1, n M]$. It is calculated according to

$$
\mathrm{p}_{\mathrm{n}}=\frac{\left.\sum_{i=(n-1) M+1}^{n M}\right\}_{i}}{M}, n=1,2, \ldots
$$

where,

$$
Y_{i}=\left\{\begin{array}{lll}
1 & \text { for } & \hat{a}_{i} \neq a_{i} \\
0 & \text { for } & \hat{a}_{i}=a_{i}
\end{array}\right.
$$

The performance of an equalizer is evaluated by the help of the convergence time $T$ and the residual mean square error $\mathrm{E}_{\mathrm{T}} \mathrm{T}_{\mathrm{c}}$ is considered as the $E_{i m e}$ after which $\epsilon_{n}$ fluctuates around 
a constant value and $E$ is the average value of $\varepsilon_{n}$ after conver-
gence has been settled.

The figures 5-7 give the results of the proposed SAE with $N_{1}=$ $9, N_{0}=21$, and $\mu_{1}=5.10^{-4}$ in the case of $L=8$. Fig. 5 shows the defëndance of $P_{n}$ on $n$. Without equalization, $P_{n}$ is 70 percent (curve (1).). The value of $P_{n}$ when using conventional SAE ( $F_{2}$ only) is shown in curve (2) while that of the proposed SAE scheme $\left(\mathrm{F}_{1}+\mathrm{F}_{2}\right)$ is shown in curve (3). It is clear that the cenvergence the conventional SAE (curve (2)) .

Relation between $\epsilon_{n}$ and $\pi$ for $\mu_{2}=8 \cdot 10^{-5}$ is shown in Fig. 6 . The values of. $\mathrm{E}$ and $\mathrm{T}_{c}$ for different values of $\mu_{2}$ are given in $\mathrm{Fig} .7$. From this figure it is obvious that the performance of the proposed scheme is better than that of the conventional one.

In the case of four level data $(\mathrm{L}=4)$, the results are given in the figures 8,9 and 10 ; by the same procedure explained in the previous case. It is concluded that the performance of the proposed SAE is also better than that of the conventional one.

Several trials have been done to reach the optimum values of $i$ in the cases of $L=8$ and $L=4$, those values are 600 and 100 samples respectively.

In practical realization of the proposed equalization scheme, there should be a unite for estimating the values of $i_{c}$. The implementation of such a unite is now under our study.

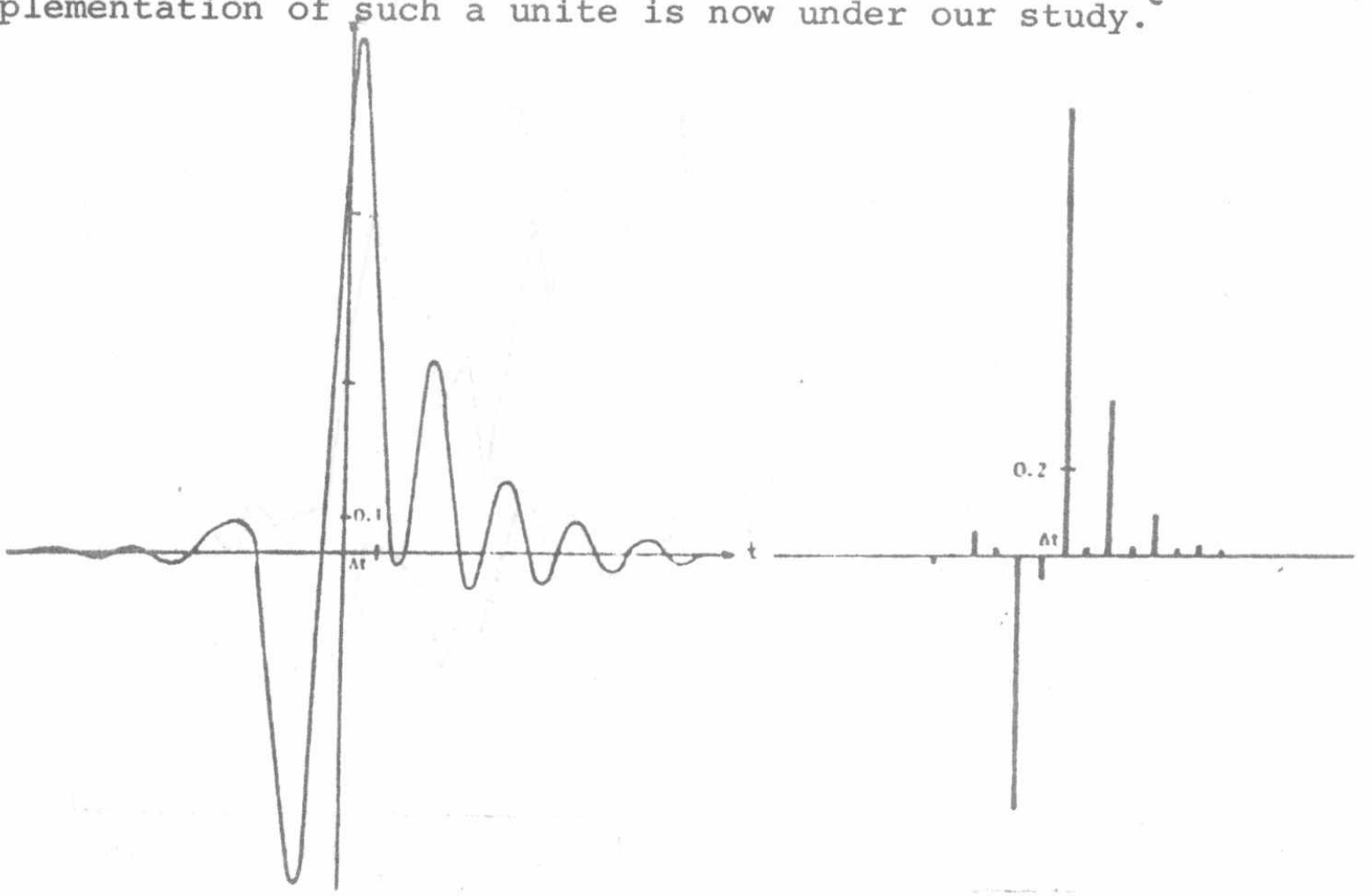

Fig. 3. Impulse response of the

Fig.4. Sampled impulse response channel 
$\Gamma \mathrm{P}_{n}$

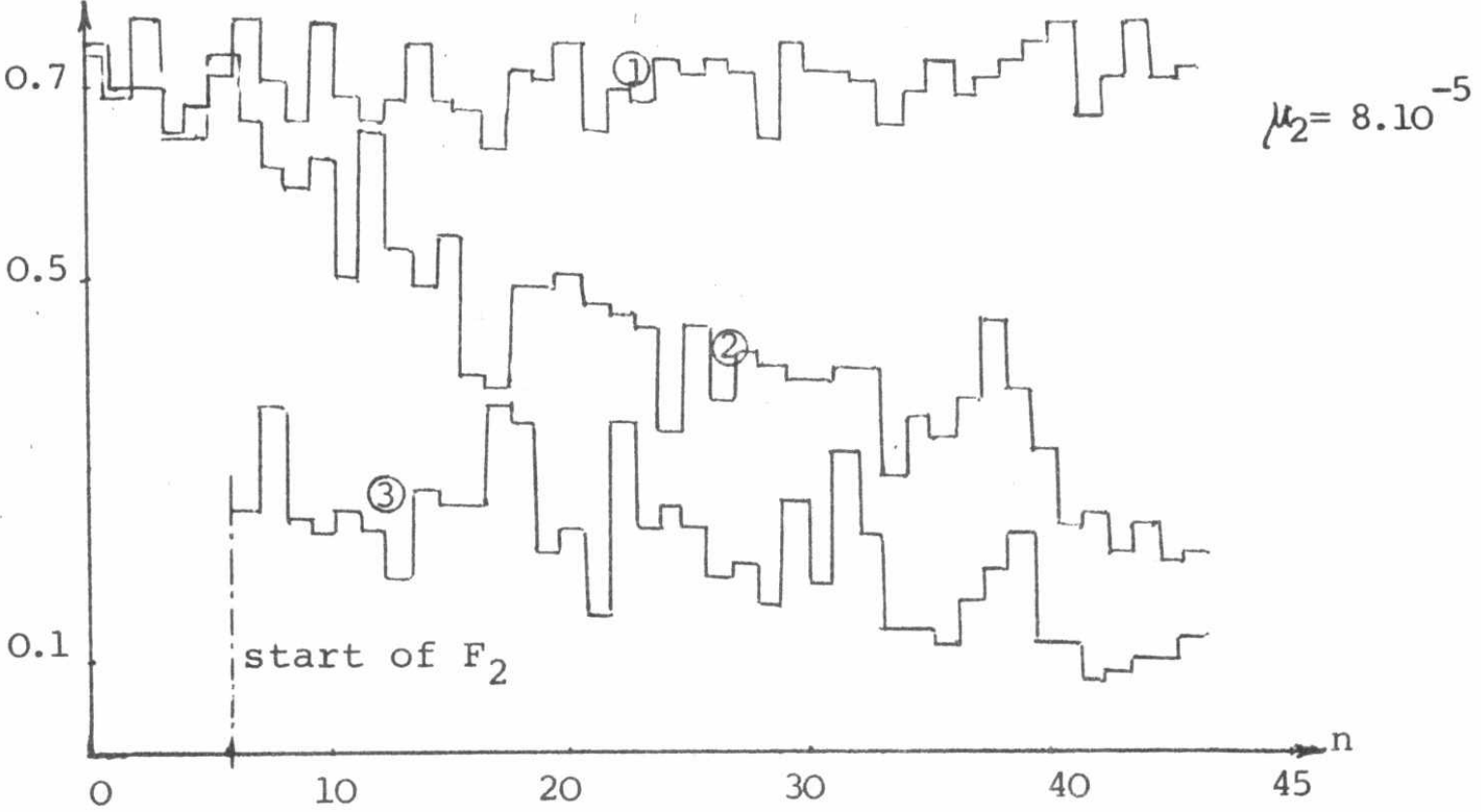

Fig. 5. Evolution of the error rate. (1) output of the channel. (2) output of the conventional equalizer $\left(F_{2}\right.$ only). (3) output of the proposed scheme. $(L=8)$.

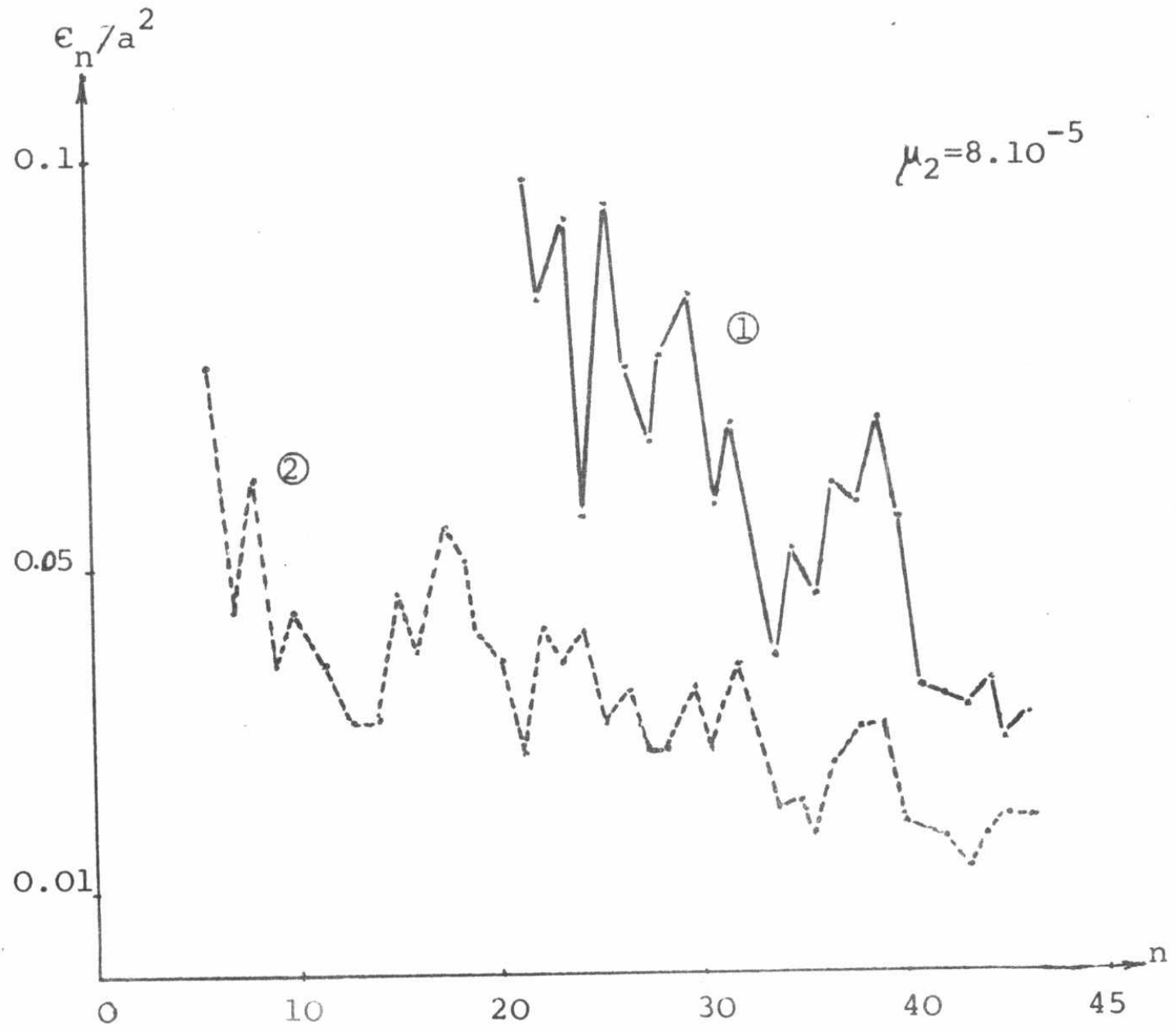

Fig.6. Evolution of mean square error. (1) output of $\mathrm{F}_{2}$ d only. (2) output of the proposed scheme $(\mathrm{L}=8)$. 


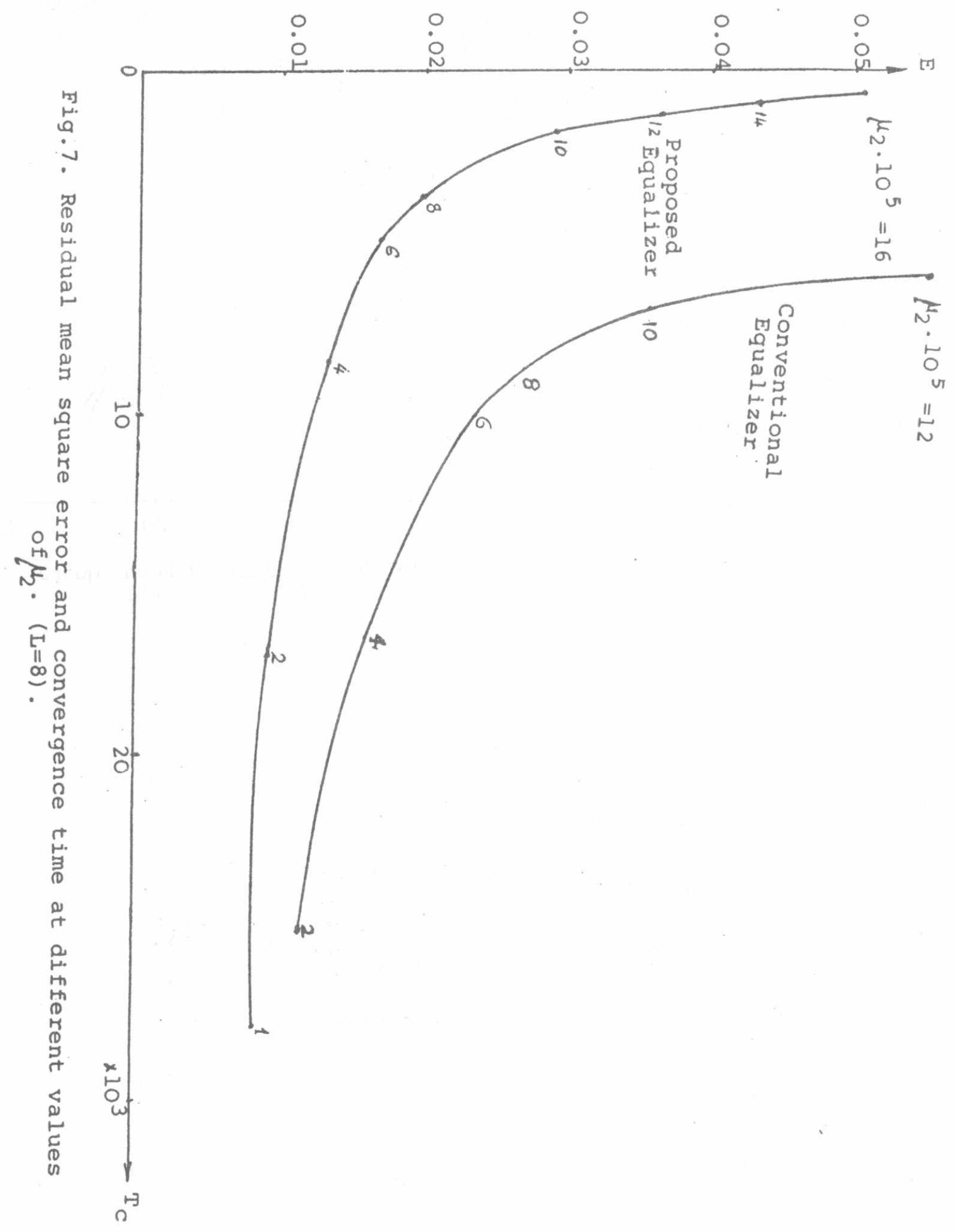


$\Gamma$

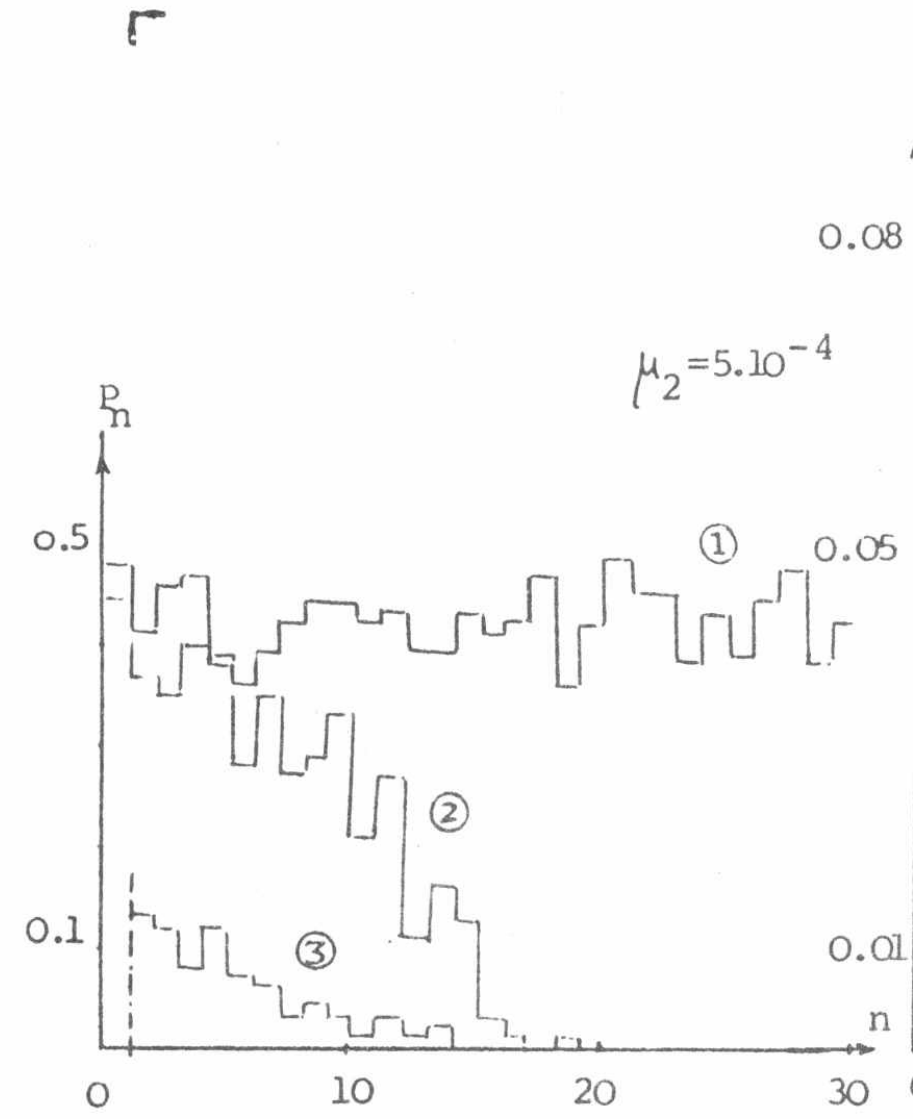

Fig. 8. Evolution of error rate. $(L=4)$.
Fig.9. Evolution of mean square error. $(L=4)$.

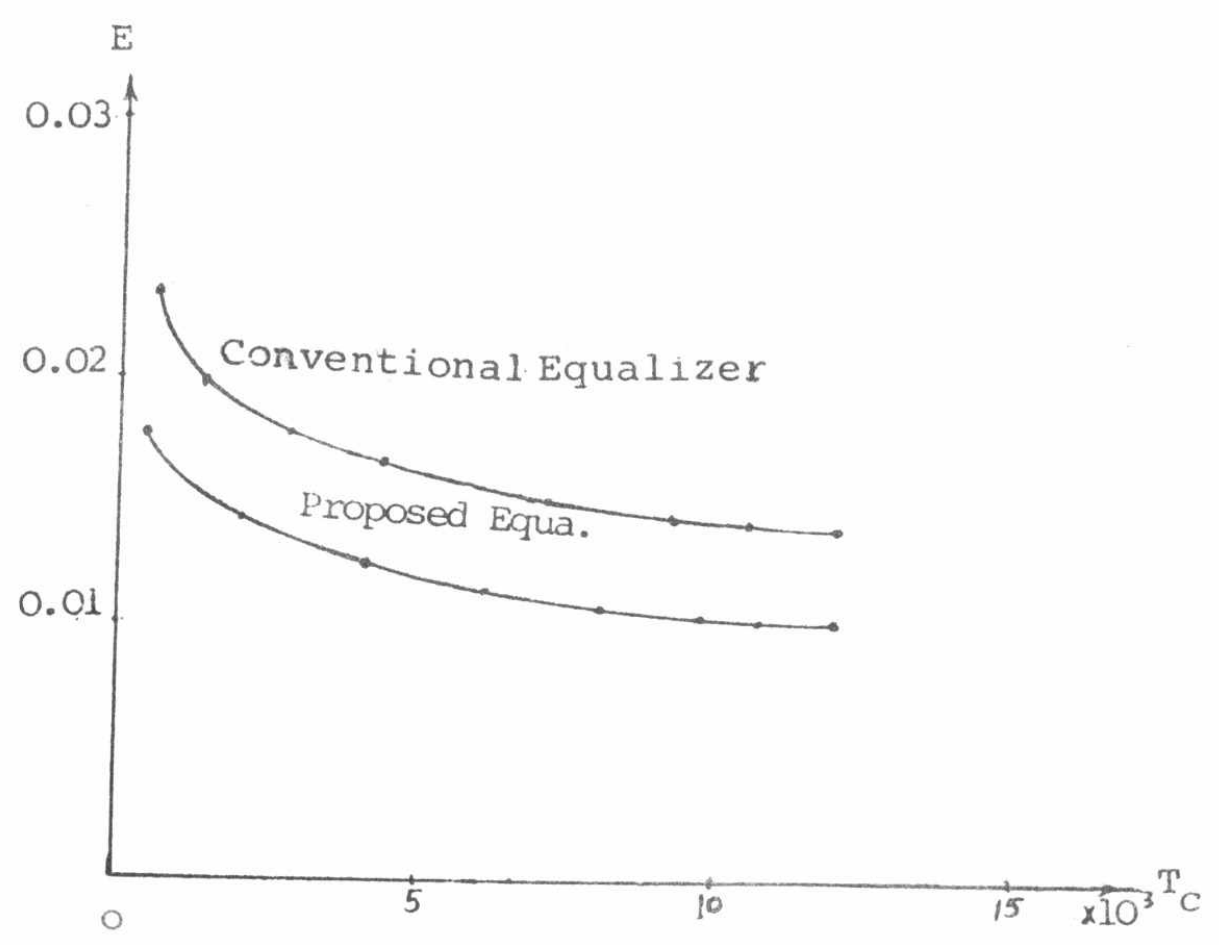

Fig. 10. Residual mean-square error and convergence time at different values of $\mu_{2} \cdot(L=4)$. 


\section{CONLCUSIONS}

In this paper we have introduced a self adaptive equalizer with rapid acquisition. The results show that this scheme has faster speed of convergence and smaller residual mean square error than the conventional self adaptive equalizer. For example (see Fig.7), the proposed self adaptive equalizer ensures a steady state mean square output error of the value 0.012 with a convergence time of 2800 baud intervals. The conventional self adaptive equalizer that ensures the same steady state error needs 11000 baud intervals to converge.

The proposed scheme does not require more computing power than the conventional self adaptive equalizers. That is due to the fact that the two adaptive filters, of which the proposed self adaptive equalizer consists, are updated sequentially.

\section{RBFERENCES}

1. J.G.Proakis "Advances in equalization for intersymbol interference" in Advances in Communication Systems, Vol.4, New York : Academic Press, 1975, pp. 124-194.

2. B. Widrow and J.M. McCool "A comparision of adaptive algorithms based on the methods of steepest descent and random search," IEEE Trans. on Antennas and propagation, Vol. Ap-24, No. 5, Sept. 1976.

3. Y. Sato, "A method of self-recovering equalization for multilevel amplitude modulation systems", IEEE Trans. Comm., Vol. COM-23, Johe 1975, pp. 679-682.

4. D.N. Godard "Self-recovering equalization and carrier tracking in two-dimensional data communication systems", IEEE Trans. Comm., Vol. COM-28, Nov. 1980, pp. 1967-1975.

5. A. Benvensites, M. Goursat, and G.Ruget, "Robust identification of a nonmintmum phase system : blind adjustment of a linear equalizer in data communication", IEEE Trans. Automatic Contr., Vol. AC-25, June 1980, pp. 385-399.

6. O.Macchi and E. Eweda, "Convergence analysis of selfadaptive equalizers", IEEE Trans. Inform. Theory, Vol. IT-30, No. 2, March 1984, pp. 161-175.

7. J.E. Mazo "Analysis of decision-directed equalizer convergence" BSTJ, Dec. 1980, pp.1857-1976.

8. B.Widrow \& S.D. Stearns "Adaptive signal processing" Printice-Hall, Inc., Englewood chiff, New Jersey, 1985.

9. Shahidu H. Qureshi " Adaptive Equalization" Proceeding of the IEEE, Vol. 73, No. 9, Sept. 85 . 